\title{
BMJ Open Evaluation of the teaching recovery techniques community-based intervention for accompanied refugee children experiencing post-traumatic stress symptoms (Accompanied refugeeS In Sweden Trial; ASsIST): study protocol for a cluster randomised controlled trial
}

Georgina Warner (D , ${ }^{1}$ Natalie Durbeej, ${ }^{1}$ Raziye Salari, ${ }^{1}$ Karin Fängström, ${ }^{1}$ Elin Lampa, ${ }^{1}$ Zaruhi Baghdasaryan, ${ }^{1}$ Fatumo Osman, ${ }^{1}$ Sandra Gupta Löfving, ${ }^{1}$ Anna Perez Aronsson, ${ }^{1}$ Inna Feldman, ${ }^{1}$ Filipa Sampaio (1) , ${ }^{1}$ Richard Ssegonja, ${ }^{1}$ Anna Bjärtå, ${ }^{2}$ Elisabet Rondung, ${ }^{2}$ Anna Leiler, ${ }^{2}$ Elisabet Wasteson, ${ }^{2}$ Rachel Calam, ${ }^{3}$ Brit Oppedal, ${ }^{4}$ Brooks Keeshin, ${ }^{5}$ Anna Sarkadi ${ }^{1}$

To cite: Warner G, Durbeej N, Salari R, et al. Evaluation of the teaching recovery techniques community-based intervention for accompanied refugee children experiencing post-traumatic stress symptoms (Accompanied refugeeS In Sweden Trial; ASsIST): study protocol for a cluster randomised controlled trial. BMJ Open 2020;10:e035459. doi:10.1136/ bmjopen-2019-035459

- Prepublication history for this paper is available online. To view these files, please visit the journal online (http://dx.doi. org/10.1136/bmjopen-2019035459).

\section{Received 06 November 2019} Revised 06 February 2020 Accepted 29 April 2020

Check for updates

(c) Author(s) (or their employer(s)) 2020. Re-use permitted under CC BY-NC. No commercial re-use. See rights and permissions. Published by BMJ.

For numbered affiliations see end of article.

Correspondence to Dr Georgina Warner; georgina.warner@pubcare.uu.se

\section{ABSTRACT}

Background Refugee children have often experienced traumas and are at significant risk of developing mental health problems, such as symptoms of post-traumatic stress disorder (PTSD), depression and anxiety, which can continue for years after resettlement. The Accompanied refugeeS In Sweden Trial (ASsIST) aims to evaluate a community-based intervention, called 'Teaching Recovery Techniques' (TRT), for accompanied refugee minors experiencing PTSD symptoms.

Methods/design A cluster randomised controlled trial will be conducted in which participants will be randomly allocated to one of the two possible arms: the intervention arm $(n=113)$ will be offered the TRT programme and the waitlist-control arm $(n=113)$ will receive services as usual, followed by the TRT programme around 20 weeks later. Outcome data will be collected at three points: pre-intervention (T1), post-intervention (T2; C.8 weeks after randomisation) and follow-up (T3; c.20 weeks after randomisation).

Ethics and dissemination Ethical approval was granted by the Regional Ethical Review Board in Uppsala (Ref. 2018/382) $\left(24^{\text {th }}\right.$ February 2019). Results will be published in scientific journals.

Trial registration details ISRCTN17754931.

Prospectively registered on $4^{\text {th }}$ June 2019.

\section{INTRODUCTION}

The impact of forced migration due to war and conflict is one of the most pressing worldwide issues. In 2015, over 70000 children fled to Sweden. ${ }^{1}$ Although the number
Strengths and limitations of this study

- AccompaniedrefugeeS In Sweden Trial (ASsIST) will evaluate the effectiveness of a group intervention for accompanied refugee children reporting symptoms of post-traumatic stress, called Teaching Recovery Techniques, in real-world settings across Sweden.

- The cluster design allows for the effectiveness of the intervention to be assessed in a target population from which patient and public involvement representatives have expressed the unacceptability of randomisation at the individual level. Individual randomisation was likened to the 'Iottery of asylum' and it was more favourable to be randomised as a group.

- An internal pilot will assess the feasibility of both trial recruitment plans and the proposed intervention with this group.

- The cost-utility and cost-effectiveness of the intervention will be estimated.

- Given the age range and diversity of backgrounds, there is potential for heterogeneity of treatment effect. Subgroup analysis will explore differential effects and inform clinical decision-making.

of new arrivals has fallen and Sweden is not facing the same challenge as a few years ago, there is still a significant number of refugee children requiring support, protection and care. We know that children exposed to war are at an increased risk of developing mental health problems, in particular, symptoms 
of post-traumatic stress disorder (PTSD). An international meta-analysis, including studies conducted during an ongoing conflict up to 5 years after the end of a conflict, reported a pooled PTSD prevalence estimate of $47 \% .^{2}$ Such mental health issues, particularly PTSD, can be exacerbated by factors associated with displacement including detainment. ${ }^{3}$ Migration status itself can be considered a risk factor for children's mental health, particularly for internalising problems. ${ }^{4}$ Despite this elevated risk, there is a relative paucity of literature on mental health interventions for refugee children. Accompanied refugee children, in particular, are largely overlooked in research studies where the focus generally lies with the needs of unaccompanied minors. ${ }^{5-8}$ A recent study of accompanied children in a reception centre in Germany identified PTSD among $33 \%$ of children aged 7 to 14 years, ${ }^{9}$ indicating a shared need among this group. A quasi-experimental evaluation of Teaching Recovery Techniques (TRT), a psychosocial group intervention to reduce symptoms of post-traumatic stress, conducted in Norway included a small number of accompanied children and demonstrated promising results with this group. ${ }^{10} \mathrm{~A}$ stronger evidence base of what works to help alleviate symptoms of mental ill health and what aid in successful integration for these children is required.

TRT was developed by the Children and War Foundation in the UK and Norway to enhance coping skills and promote recovery from post-traumatic stress among children aged 8 or above exposed to armed conflicts or natural disasters. ${ }^{11} 12$ The idea was to design an intervention that could be cost-efficient and applicable in lowresource settings, where large numbers of children are in need of intervention with a shortage of professionals who could offer support.

The effectiveness of TRT has been examined in multiple studies across war-affected countries as well as countries hosting refugee children, including Sweden, and positive effects have been reported. In three randomised controlled trials (RCTs) in Palestine and Gaza, children who received TRT showed fewer post-traumatic, depressive, distress and grief symptoms and increased psychosocial well-being. ${ }^{13-15}$ Another RCT conducted in Australia reported similar findings for TRT in terms of reduced symptoms of depression in children, but no change in symptoms of post-traumatic stress or internalising and externalising problems. ${ }^{16}$ In two quasi-experimental studies in Norway $^{10}$ and Sweden, ${ }^{6}$ children reported fewer symptoms of post-traumatic stress and depression and improved psychosocial well-being after participating in TRT. Exploration of participants' and parents' perceptions of TRT indicate the programme is well-accepted. ${ }^{6}{ }^{16}$ The Accompanied refugeeS In Sweden Trial (ASsIST) aims to further strengthen the evidence base of TRT, specifically for accompanied refugee children aged 8 to 17 years residing in Sweden. This paper outlines the protocol for ASsIST.

\section{OBJECTIVES}

The objectives of the trial are:

1. To evaluate whether the TRT programme has an effect on accompanied refugee child mental health, specifically symptoms of post-traumatic stress, anxiety and depression, in comparison to similar children who only receive services as usual.

2. To evaluate whether the TRT programme has an effect on accompanied refugee child self-efficacy and well-being, which relate to the programme theory of change.

3. To identify which subgroups report the most/least benefit of TRT with regard to symptoms of post-traumatic stress, anxiety and depression; self-efficacy; and wellbeing.

4. To describe the extent to which TRT is implemented with fidelity to programme design.

5. To estimate the cost-utility and cost-effectiveness of the TRT programme.

It is hypothesised that, when compared with children who have not received the intervention (the waitlistcontrol arm), children who have received TRT (the intervention arm) will demonstrate fewer self-reported/ parent-reported symptoms of mental ill-health, specifically PTSD, depression and anxiety symptoms. It is further hypothesised that, when compared with the waitlist-control arm, the intervention arm will report greater self-efficacy and well-being. In view of the anticipated health benefits of the intervention that could result in less contact with healthcare and increased productivity, the intervention is also expected to be cost-effective compared with the wait-list control.

\section{METHODS AND ANALYSIS \\ Design}

A two-arm cluster randomised waitlist-control superiority trial will be conducted to evaluate the effectiveness of the TRT programme in improving mental health outcomes in accompanied refugee children who have parent-reported (child age: 8 to 11 years) or selfreported (children age: 12 to 17 years) symptoms of PTSD. The design was informed by an ongoing RCT with unaccompanied refugee youth in Sweden, to aid comparability across the evaluations. ${ }^{17}$ The intervention arm will be offered the TRT programme immediately after randomisation and the waitlist-control arm around 20 weeks later; both trial arms will have access to services as usual. Assessments will take place at three points: pre-intervention (T1), post-intervention (T2; c. 8 weeks after randomisation) and follow-up (T3; c.20 weeks after randomisation). T2 will evaluate immediate effects of TRT directly after the intervention and T3 will evaluate the maintenance of effects or delayed effects. T3 has been set at 20 weeks so the waitlist control group can receive TRT in a timely manner (see figure 1 for an overview of assessments.) 


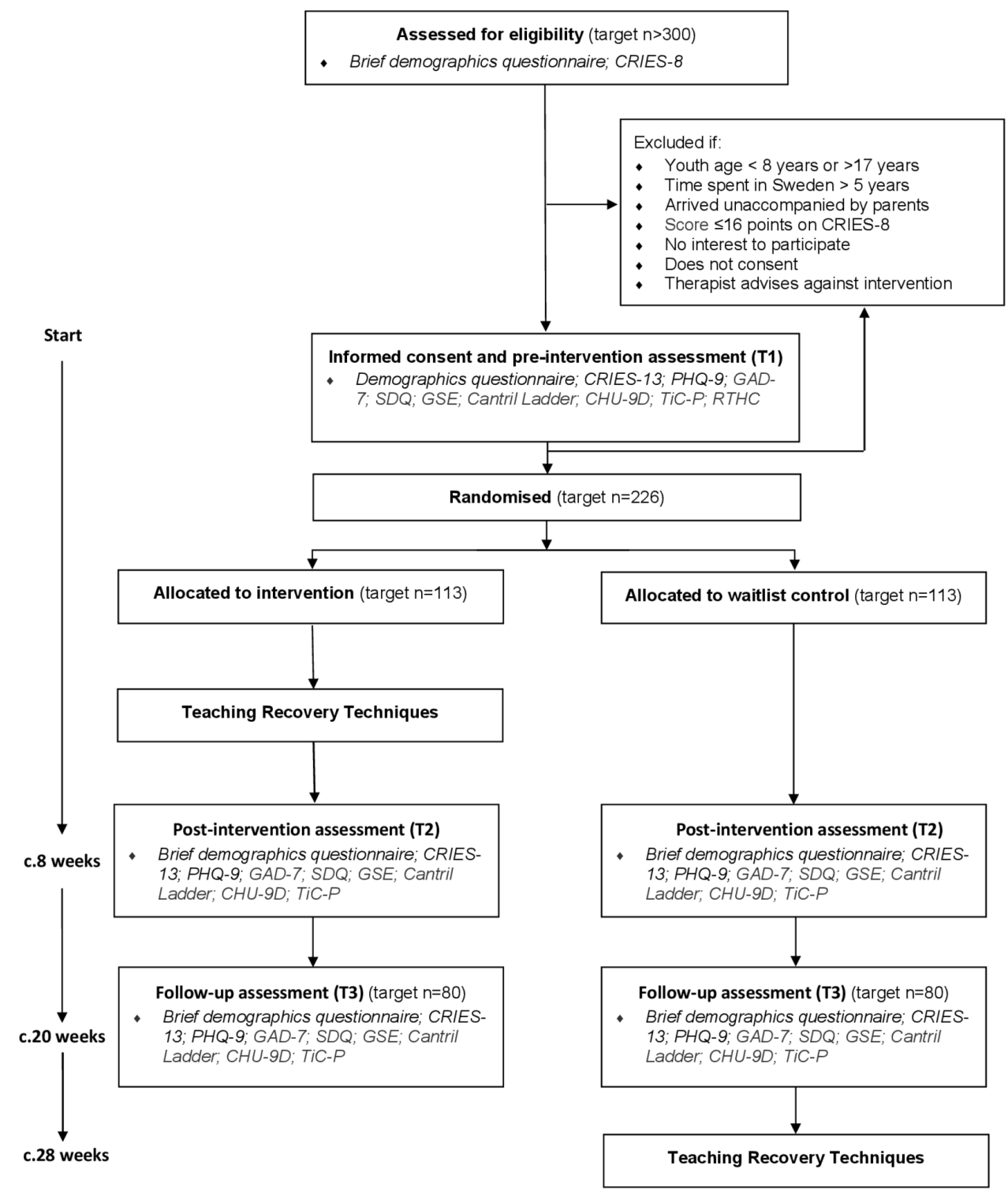

Figure 1 AccompaniedrefugeeS In Sweden Trial (ASsIST) flow chart. CHU-9D,Child Health Utility 9D; CRIES-8/CRIES13,Children's Revised Impact ofEvents Scale; GAD-7, Generalised AnxietyDisorder-7; GSE, General Self Efficacy; PHQ-9,

Patient Health Questionnaire-9; RTHC, Refugee Trauma History Checklist; SDQ,Strengths and Difficulties Questionnaire; TiC-P, Trimbos Costsassociated with Psychiatric Illness.

\section{Setting}

TRT-trained 'group leaders' will deliver the intervention; two group leaders deliver each group (with the assistance of an interpreter, if necessary). The groups will be delivered in a range of community settings (eg, schools, healthcare centres, non-governmental organisations (NGOs)) across Sweden. Sites include both urban and rural municipalities that have accepted to host refugees. To promote intervention stability it is recommended that each site has an assigned local coordinator and at least one experienced group leader (ie, who has conducted at least two previous TRT groups). Supervision will be offered to TRT group leaders. Assessments for the RCT will also take place in community settings.

\section{Participants}

Children are eligible to participate if all of the following criteria are satisfied at the time of randomisation:

- The child is aged 8 to 17 years old.

- The child has spent 5 years or less in Sweden.

- The child arrived in Sweden accompanied by family.

- The child screens positive on the Children's Revised Impact of Event Scale (CRIES-8) PTSD screening tool ( $\geq 17$ points).

- The child is interested to participate in a group intervention.

- The legal guardian (8 to 14 years) or child (15 to 17 years) consents to participation.

- There is no ongoing treatment where a therapist advises against participation. 


\section{Recruitment}

Children (boys and girls) will be referred to the trial by community workers (eg, school staff, nurses, NGO staff members) who have concerns about the child's mental health. Parents and children can also self-refer. The CRIES- $8^{18}$ will be used by the trial team to identify children with symptoms of PTSD. Those with scores $\geq 17$ will be eligible to participate in the study. The Child Health and Parenting (CHAP) research group at Uppsala University has established relationships with community sites with TRT-trained staff across Sweden (eg, Huddinge; Linköping; Uppsala; Östersund; Stockholm; Västerås). New sites will be approached during the trial period. Where possible, the research team will work with Barnens Rätt i Samhället (BRIS), who are responsible for TRT training in Sweden, to facilitate the matching of experienced leaders to new sites to deliver alongside newly trained personnel. It is anticipated that around 10 sites will recruit to the trial. Communications about the research study will be distributed to community sites directly by CHAP and posted online on the CHAP website. In an exploratory study with unaccompanied refugee minors, $90 \%$ of those screened for participation met the cut-off on the CRIES- $8 .{ }^{6}$ The treatment retention rate was $59 \%$, with most dropouts occurring right before or just after the start of the group. To take these factors into account, an over-recruitment is planned for the present project.

Children who meet the inclusion criteria will be invited to a group 'information and assessment' meeting with their parent(s). Written informed consent and T1 measures will be collected at the meeting prior to randomisation on site. The child being interested in participating is an inclusion criteria for the trial. This is the case for all participants including children under 15 years of age, who will be asked to give their assent to take part. The informed assent/consent relates only to the ASsIST study; no ancillary studies are planned. No biological specimens will be collected as part of the ASsIST study.

Several strategies designed to minimise the level of attrition from the trial will be put in place. First, efforts will be made during the consent process and via information leaflets to make sure participants are fully aware of what the research study involves and what will be expected from them, and to emphasise the value of taking part in the study. Second, the trial has been branded the ASsIST project (Accompanied refugeeS In Sweden Trial). ASsIST will be communicated in a professional and attractive way that participants will be likely to identify with and be interested in. For example, advertisements will be written in youth-friendly language and translated into the various languages of anticipated participants. This process will be informed by our patient and public involvement activity with Refugee Advisors, who will also provide broader recruitment support to the study. Third, families will be offered a small monetary incentive (shopping vouchers to the value of a cinema ticket) for each of the three data collection sessions to compensate for their time spent in completing the questionnaires. Finally, CHAP will work to keep community contacts engaged through regular email and telephone correspondence, as well as providing support in making referrals to the project.

\section{Sample size}

Recruitment of 226 eligible children to the project will allow detection of an effect size of 0.5 at $\mathrm{p}<0.05$ with $80 \%$ power. This allows for an estimated intraclass correlation coefficient of 0.05 and a study dropout rate of up to $41 \%$, as informed by an exploratory study. The minimum required sample is 160 participants.

\section{Randomisation}

A computer-generated randomisation sequence will be used to assign the clusters to the intervention and waitlistcontrol arms in a 1:1 ratio. Block randomisation of block sizes 4,6 or 8 will be generated in a computerised randomisation schedule. Randomisation will take place after T1 data collection. The allocation sequence will be concealed using an online central randomisation service set up and maintained by a professional third party (www.sealedenvelope.com) that will conceal the sequence until group assignment. The randomisation process will require the research team to $\log$ into a password-protected website and enter the cluster ID code in order to receive the allocation.

\section{Blinding}

Randomisation will take place in the community, at scheduled group 'information and assessment' meetings, directly after T1 data collection. The research team will oversee the randomisation process. Participants will not be blinded to group allocation; they will be informed of group allocations immediately at the group meeting, along with the assigned TRT group leaders. Allocation status will be recorded on a secure online platform ( www.sealedenvelope.com). Data collection will not be blinded; however, as the outcome data are collected using self-completion questionnaires rather than through observation or interview, outcome data is less susceptible to information bias and interview effects. ${ }^{19}$ Given the participants nor group leaders are blinded, there is no requirement for an unblinding procedure. Outcome data spreadsheets will use anonymous participant identity numbers; however group status will be apparent due to the inclusion of attendance data for the intervention group.

\section{Control arm}

Children assigned to the waitlist-control arm will receive services as usual because the aim of the trial is to determine whether the TRT programme provides added value. The offer is likely to include school health services and contact with their general practitioner. Other services are unlikely to be highly similar to the TRT programme, as reconnaissance suggests that typically few group therapy programmes are available for accompanied refugee children. Any services that children do receive, including other therapy programmes, will be captured in the 
Trimbos/institute for Medical Technology Assessment (iMTA) Questionnaire for Costs associated with Psychiatric Illness (TiC-P) (see Other measures section).

\section{Intervention arm}

The intervention arm will receive the Swedish translation of the TRT programme. The same manual is applicable to the full age range of the study (8 to 17 years). The intervention is based on the principles of traumafocussed cognitive behavioural therapy (TF-CBT), agreed to be the method of choice for treating PTSD in children and adolescents, ${ }^{20}$ but is delivered by professionals who have received a brief training (not full TF-CBT certification). The group-based cognitive-behavioural programme includes five youth sessions and two caregiver sessions. Child sessions focus on teaching the children about symptoms that may occur after experiencing a traumatic event and giving them strategies to reduce symptoms of intrusive memories, hyper arousal and avoidance. They incorporate the following components of TF-CBT: psychoeducation, affective modulation skills, cognitive coping and processing, trauma narrative, in vivo mastery of trauma reminders and future development. Caregiver sessions focus on teaching the caregivers about the symptoms their children might be experiencing and describing the content of the child sessions. The caregivers are also instructed in how to support the children through listening and comforting, when needed, as well as through maintaining routines and activities. Furthermore, the caregivers receive information on how to seek care if the child needs additional help after TRT. The caregiver sessions are delivered without the children, in parallel with the first two child sessions. In Sweden, a 'getting to know each other session' is offered prior to the core TRT sessions and a 'follow-up session', to consolidate learning and give participants the opportunity to talk about their experience of taking part in the programme, is offered afterwards. Sessions will be delivered over seven consecutive weeks. Each session will last 2 hours (including a break). TRT group leaders receive 3 days of training in programme delivery from BRIS. Two group leaders deliver each group (with the assistance of an interpreter, if necessary).

\section{Participant timeline}

A schematic diagram of the participant timeline can be found in figure 1. Children are screened for eligibility, which could be done individually or at a group 'information and screening' meeting. For eligible children, informed consent and $\mathrm{T} 1$ assessments take place at a group 'information and assessment' meeting. A case will be randomised once all pre-intervention data has been collected. Follow-up data will be collected from all participants at scheduled group meetings at two points: first (T2), c.8 weeks ( \pm 1 week) after randomisation (equivalent to end of TRT programme delivery) and second (T3), c.20 weeks ( \pm 2 weeks) after randomisation (equivalent to c.3 months after TRT programme delivery).

\begin{tabular}{lclc} 
Table 1 & Overview of measures & & \\
\hline Measure & $\begin{array}{l}\text { Child (8- } \\
\text { 11 years) }\end{array}$ & $\begin{array}{l}\text { Child (12- } \\
17 \text { years) }\end{array}$ & Parent \\
$\begin{array}{l}\text { Demographics } \\
\text { Questionnaire }\end{array}$ & $\checkmark$ & $\checkmark$ \\
CRIES-13 & $\checkmark$ & $\checkmark$ & $\checkmark$ \\
PHQ-9 & & $\checkmark$ & $\checkmark$ \\
GAD-7 & & $\checkmark$ & $\checkmark$ \\
SDQ & & $\checkmark$ & $\checkmark$ \\
GSE & $\checkmark$ & \\
Cantril Ladder & $\checkmark$ & $\checkmark$ & \\
CHU-9D & $\checkmark$ & $\checkmark$ & \\
TiC-P & & $\checkmark$ & $\checkmark$ \\
RTHC & & $\checkmark$ & $\checkmark$
\end{tabular}

CHU-9D, Child Health Utility 9D; CRIES-8/CRIES-13, Children's Revised Impact of Events Scale; GAD-7, Generalised Anxiety Disorder-7; GSE, General Self Efficacy; PHQ-9, Patient Health Questionnaire-9; RTHC, Refugee Trauma History Checklist; SDQ, Strengths and Difficulties Questionnaire; TiC-P, Trimbos Costs associated with Psychiatric IIIness.

\section{Outcome measures}

The study will primarily measure changes in parentreported (child age: 8 to 11 years) or self-reported (child age: 12 to 17 years) child mental health, specifically symptoms of PTSD, depression and anxiety. A combination of primary mental health measures is being used due to the complex trauma that can be experienced by the children. Apart from adverse events before and during migration, the asylum and resettlement process per se involves stressors. Complex trauma can lead to social difficulties, behavioural and emotional symptoms, psychosomatic problems, and sleep problems as well as PTSD symptoms. ${ }^{21-23}$

Secondary assessments will include measures of emotional and behavioural difficulties, self-efficacy and well-being, which relate to the TRT programme theory of change. All outcome measures will initially be available in Swedish, English, Arabic, Dari, Farsi, Somali and Tigrinya, with other languages made available if necessary. Where possible, validated translations will be used (eg, CRIES, PHQ-9, GAD-7, SDQ). However, for particular languages (eg, Tigrinya) and measures (eg, RTHC, CHU-9D, TiC-P) the native language skills of the CHAP research group will be used to produce translations. The measures will be administered at T1, T2 and T3. See table 1 for an overview of measures by respondent (child 8 to 11 years; child 12 to 17 years; parent). The specific metrics, methods of aggregation and time points for the outcomes are described in the statistical analyses section.

Children's Revised Impact of Events Scale (CRIES-13/CRIES-8)

The CRIES- $13^{17}$ is a 13 -item measure of PTSD symptoms. Individual items are rated according to the frequency 
of their occurrence during the past week (None $=0$, Rarely $=1$, Sometimes $=3$ and A lot $=5$ ) and in relation to a specific traumatic event. Scores are obtained for four intrusion items (eg, Do you think about it even when you don't mean to?), four avoidance items (eg, Do you try not to talk about it?) and five arousal items (eg, Do you get easily irritable?). Total scores on the scale range from 0 to 65 with a cut-off score of 30 or above. The total score has shown to have good internal consistency, and to successfully categorise over $75 \%$ of children with and without a PTSD diagnosis. ${ }^{17} 24$ CRIES-8 includes the intrusion and avoidance items. The factor structure and internal consistency of the CRIES-8 (Cronbach's $\alpha=0.75$ ) was confirmed in a study of asylum-seekers in Uppsala, Sweden. ${ }^{25}$ In the present study, the CRIES- 8 will be used to screen the children (eligibility cut-off of $\geq 17$ ) and the CRIES-13 will be used as a primary outcome measure.

\section{Patient Health Questionnaire-9}

The Patient Health Questionnaire-9 (PHQ-9) ${ }^{26}$ is a 9-item instrument for screening, diagnosing, monitoring and measuring severity of depression. Individual items (eg, Little interest or pleasure in doing things) are rated according to the frequency of their occurrence during the past 2 weeks (Not at all $=0$, Several days $=1$, More than half the days $=2$, Nearly every day $=3$ ). Total scores on the scale range from 0 to 27 with cut-off scores of 5, 10, 15 and 20 for mild, moderate, moderately severe and severe symptoms, respectively. The instrument has shown high internal consistency (Cronbach's $\alpha=0.86$ and 0.89 in two primary care samples, respectively) and test-retest reliability $(\mathrm{r}=0.84) .{ }^{26}$ Both construct validity and diagnostic validity for major depression has been established in several studies, and sufficient sensitivity ( 0.71 to 0.87 ) and high specificity (0.88 to 0.95 ) has been found for PHQ-9 $\geq 10 .{ }^{27}$ PHQ-9 has also shown responsive in measuring treatment outcomes, and a change in scores of 5 has been suggested to reflect a clinically relevant change. ${ }^{28}$ It has been successfully tested with adolescents ${ }^{29}$ and will be administered as a self-report measure for children aged 12 years and above in the present study. A parent-report version, often used in clinical settings, will be used with children under 12 years old.

\section{Generalised Anxiety Disorder-7}

The Generalised Anxiety Disorder-7 (GAD-7) ${ }^{30}$ is a 7 -item measure originally developed to screen for generalised anxiety disorder. It has, however, also frequently been used to assess severity of more general anxiety symptoms. ${ }^{31}$ Individual items (eg, Feeling nervous, anxious or on edge) are rated according to the frequency of their occurrence during the past 2 weeks (Not at all $=0$, Several days $=1$, More than half the days $=2$, Nearly every day $=3$ ). Total scores on the scale range from 0 to 21 with cut-off scores of 5, 10 and 15 for mild, moderate and severe symptoms, respectively. It has shown high internal consistency (Cronbach's $\alpha=0.92$ ) and seems to function well as an indicator of symptom severity. ${ }^{29}$ The GAD-7 has been successfully tested with adolescents ${ }^{32}$ and will be administered as a self-report measure for children aged 12 years and above in the present study. A parent-report version, often used in clinical settings, will be used with children under 12 years old.

\section{The Strengths and Difficulties Questionnaire}

The Strengths and Difficulties Questionnaire $(\mathrm{SDQ})^{33}$ is a 25-item measure of child emotional and behavioural difficulties. Individual items (eg, Often has temper tantrums or hot tempers in the parent version and I get very angry in the child version) are rated according to how true the statement is for the child (Not True $=0$, Somewhat True $=1$, Certainly True=2). The 25 items are divided between five scales: emotional symptoms (five items); conduct problems (five items); hyperactivity/inattention (five items); peer relationship problems (five items); and prosocial behaviour (five items). Total scores are generated from 20 items taken from the first four subscales and range from 0 to 40 , with a higher score indicating more emotional and behavioural difficulties. The total scale has good internal consistency, with Cronbach's $\alpha$ of 0.80 or greater. ${ }^{34}$

\section{General Self Efficacy scale}

The General Self Efficacy (GSE) $)^{35}$ scale is a 10-item measure that assesses the strength of individuals' believes in their own ability to respond to difficult situations and to deal with obstacles or setbacks. Individual items (eg, I can always manage to solve difficult problems if I try hard enough) are rated according to how true the statement is for that individual $(1=$ Not at all true, 2=Hardly true, 3=Moderately true, $4=$ Exactly true). Total scores range from 10 to 40 with a higher score indicating more self-efficacy. In samples from 25 nations, Cronbach's $\alpha$ ranged from 0.75 to 0.91 , with the majority in the high $0.80 \mathrm{~s}{ }^{36}$

\section{The Cantril Ladder}

The Cantril Ladder ${ }^{37}$ measures well-being and life satisfaction. The respondent is presented with a picture of a ladder numbered from 0 to 10 , with the following text: "Here is a picture of a ladder. Suppose the top of the ladder represents the best possible life for you and the bottom of the ladder the worst possible life. Where on the ladder do you feel you stand at the present time? "Scores range from 0 to 10 with a higher score indicating greater well-being and life satisfaction. A score of 4 or below is indicative of 'suffering' and 7 or above 'thriving'. The scale has proven a valid measure of general psychosocial health among children/youth ages 10 to 17 years. ${ }^{38}$ The Cantril Ladder will be administered at each TRT session to inform a safety protocol. The score will be used as a secondary outcome, assessed at T1, T2 and T3.

\section{Other measures}

Basic demographic information will be collected for all participants. Health-related quality of life and service consumption will be measured to inform the economic evaluation. A suicidality screening tool will be used as part of a safety protocol for participants who indicate they 
have had thoughts they would be better off dead (ninth item on PHQ-9) or 'suffering' on the Cantril Ladder (ie, a score of 4 or below).

\section{Demographics questionnaire}

The study will use a short questionnaire to gather demographic information about the child and their family. It includes variables such as child's age, gender, ethnicity, time spent in Sweden and asylum status. These data will be used to describe the sample, examine the extent to which demographic characteristics are balanced between trial arms, carry out attrition analyses (ie, the extent to which participants who drop out from the intervention and waitlist-control arms are different on variables such as gender and ethnicity) and identify subgroups. The demographics questionnaire will be administered at T1 A brief version of the questionnaire that includes items for which the response may change (eg, asylum status) will be administered at $\mathrm{T} 2$ and $\mathrm{T} 3$.

\section{Refugee Trauma History Checklist}

The Refugee Trauma History Checklist $\left(\right.$ RTHC) ${ }^{39}$ is a measure of the occurrence of potentially traumatic experiences. It consists of $2 \times 8$ items, concerning potentially traumatic experiences that occurred before and during the respondents' flight, respectively. Results show low item non-response and adequate psychometric properties. ${ }^{39}$ These data will be used to describe the sample, examine the extent to which potentially traumatic experiences are balanced between trial arms, carry out attrition analyses (ie, the extent to which participants who drop out from the intervention and waitlist-control arms report different experiences) and identify subgroups. The RTHC will be administered at $\mathrm{T} 1$ only.

\section{Child Health Utility 9D}

The Child Health Utility 9D (CHU-9D) ${ }^{40}$ is a self-report measure of health-related quality of life. It consists of nine dimensions (worry, sadness, pain, tiredness, annoyance, school, sleep, daily routine and activities). Individual items are scored according to severity on the day from 1 (no problems) to 5 (severe problems). Originally developed for application with children aged 7 to 11 years, ${ }^{41-43}$ its practicality and validity in adolescents aged 11 to 17 years has also been demonstrated. ${ }^{44}{ }^{45}$ In this study, responses to the CHU-9D will be scored using the UK scoring algorithm (the only available European algorithm). The scoring algorithm was generated on a utility scale and ranges from 0 for the worst health state to 1.0 for the best health state. The CHU-9D will be administered at T1, T2 and T3 and will inform the economic evaluation.

\section{Trimbos/institute for Medical Technology Assessment}

\section{Questionnaire for Costs associated with Psychiatric Illness}

The TiC-P ${ }^{46}$ is a generic questionnaire for people with a psychiatric disorder, meaning that the items are not related to a target disorder. Distinguishing between healthcare consumption and productivity losses as a consequence of the target disorder and comorbidity is difficult, especially in psychiatric disorders, as patients also may have physical symptoms that are connected to the psychiatric illness. Moreover, psychiatric comorbidity is a common occurrence in psychiatric illness. The TiC-P will ask about the use of different services and absence from school/work over a time period preceding the date of the data collection. It will be administered at T1, T2 and T3 and will inform the economic evaluation. The parent version will be administered for all children and the adolescent version administered for children aged 12 years and above.

\section{Columbia-Suicide Severity Rating Scale screen version}

The Columbia-Suicide Severity Rating Scale (C-SSRS) screen version ${ }^{47}$ is a 6 -item structured interview or selfreport measure that assesses the presence and severity of suicidal ideation and behaviour. Individual items (eg, Have you wished you were dead or wished you could go to sleep and not wake up?) are rated according to presence over the past month (Yes or No). A positive response to item 3 (Have you been thinking about how you might do this?) indicates a moderate risk. A positive response to items 4 (Have you had these thoughts and had some intention of acting on them?), 5 (Have you started to work out or worked out the details of how to kill yourself? Do you intend to carry out this plan?) or 6 (Have you ever done anything, started to do anything or prepared to do anything to end your life?) indicates a high risk. A three multisite study including both adults and adolescent showed strong convergent validity with other established scales measuring suicidal ideation and attempts. ${ }^{47}$ Chronbach's $\alpha$ varied between 0.95 for intensity of suicidal ideation during the past week and 0.73 across all visits. The C-SSRS will be used as part of a safety protocol for participants who indicate they have had thoughts they would be better off dead (ninth item on PHQ-9) or 'suffering' on the Cantril Ladder (ie, a score of 4 or below). Safety protocol use will be captured on the fidelity checklist (see below). Frequency of safety protocol use will be reported.

\section{Intervention fidelity}

A fidelity-monitoring tool has been developed by the CHAP research team in association with TRT facilitators in order to promote and monitor adherence to the core design of the programme. The fidelity monitoring process will be implemented by TRT facilitators, who will share the data with CHAP for research purposes. After each TRT session, the facilitators complete a self-report adherence checklist, which captures: facilitator details (including profession and TRT experience level); number of participants; use of interpreter(s); number of people who required the safety protocol; and the range of core components delivered. Session attendance lists will also be shared with CHAP to inform individual participant dose.

\section{Data collection}

T1 data collection and randomisation is planned to take place between October 2019 and February 2021. T2 data 
collection occurs c. 8 weeks after T1 data collection, and is therefore projected to take place between December 2019 and April 2021. T3 data collection occurs c.20 weeks after $\mathrm{T} 1$ data collection and is projected to take place between March 2020 and July 2021.

Outcome data will be collected using a secure online platform (Qualtrics). It is estimated the survey will take around 15 min for the children aged 8 to 11 years and around 45 to $60 \mathrm{~min}$ for children aged 12 to 17 years and parents. The survey will be completed in a single session; participants may take a break, if required, and refreshments will be available. TRT facilitators will submit fidelity data on paper forms. Data will be exported/inputted into an SPSS file for analysis. Anonymous participant identity numbers will be used. The file will be saved on the university server, which is automatically backed up. All procedures comply with current regulations on personal data management.

\section{Statistical methods}

Pre-intervention and demographic characteristics will be summarised using means and SDs (or medians and IQRs) for continuous variables and percentages for categorical variables. A set of strategies will be employed to minimise the amount of missing data (eg, offering incentives for completing follow-ups). Reasons for dropouts for each condition will be reported. The possible impact of missing data will be examined via sensitivity analyses of augmented data sets. Modern analytical methods will be used to include both dropouts and participants with missing data in the analyses.

The primary comparison of the trial arms will use an intention-to-treat framework with participants analysed according to the trial arm they were randomised to, regardless of whether or not they received the intervention. Additionally, a compiler average causal effect analysis will be conducted. ${ }^{48}$ Regression analysis methods will be used to compare outcomes for the trial arms. The primary outcome is total score group differences on the primary mental health outcome measures (ie, CRIES13; PHQ-9; GAD-7) after programme delivery (T2). The secondary outcomes are: mental health outcome measure (ie, CRIES-13; PHQ-9; GAD-7) total scores at endpoint (T3); SDQ; GSE and Cantril ladder total scores after programme delivery and at endpoint (T2 and T3). Parent report will be taken as the primary measure for children aged 8 to 11 years at randomisation and selfreport taken as primary for children aged 12 to 17 years. If the preferred primary response is not available, the alternative will be used. For example, if a parent does not respond for an 8-year-old but the child provides a selfreport, the self-report will be included in the analysis.

For the mental health outcome measures (CRIES-13; PHQ-9; GAD-7), participants will also be classified as 'recovered', 'improved', 'unchanged' or 'deteriorated' based on the Reliable Change Index and Clinically Significant Change approach. ${ }^{49} 50$ This approach incorporates both a measure of whether the change in scores is larger than what is expected due to outcome measure reliability as well as the participant's shift from a clinical state to a non-clinical state. The proportions of classifications will be compared across the trial arms.

Fidelity to the design of the intervention will be summarised using descriptive statistics. It will be assessed in terms of the different dimensions measured (adherence and dose). A secondary analysis will be undertaken to quantify the extent to which the intervention effect on the primary outcomes is determined by participation in the intervention (number of sessions received). Further moderation analyses will examine the associations between improvement status and participants' characteristics (eg, age, gender, suicidal ideation).

For the economic evaluation, two types of analyses will be conducted: (i) a cost-utility analysis with outcomes measured in Quality Adjusted Life Years (QALYs) and (ii) a cost-effectiveness analysis with proportion of participants classified as treatment success as the outcome. Differences in outcomes and costs between the intervention and control groups over the trial period will be compared using generalised linear models, which allows the consideration of other distributions and functional forms to fit the data. ${ }^{51}$ Estimated differences in outcomes will be compared with differences in costs and presented as incremental cost-effectiveness ratios. ${ }^{52}$ The costeffectiveness ratios describe: (i) the price for one additional QALY gained, that is, one life year with full health and (ii) the price to get an additional successfully treated participant. Uncertainty around the cost-effectiveness estimates will be explored by using appropriate statistical and visual methods to aid decision-making. ${ }^{52}$

\section{Internal pilot}

Given previous pilot work has only been carried out with unaccompanied refugee minors in Sweden, ${ }^{6}$ an internal pilot will be conducted. The target $\mathrm{N}$ is 28 eligible children (14 per arm), which has been derived from theoretical optimal values based on the power calculation for the main trial. ${ }^{53}$ The primary objectives of the pilot study are to assess the feasibility of both trial recruitment plans and the proposed intervention. The success criteria for the internal pilot will be: (i) at least $50 \%$ of those screened for participation meet the cut-off $(\geq 17)$ on the CRIES-8; (ii) at least 28 eligible children are recruited in the first 3 months; and (iii) at least $50 \%$ of those randomised to the intervention attend at least one of the five core programme sessions and complete the T2 data collection. Descriptive statistics will be reported for the trial outcome measures. Adherence rates will also be described and the reasons for non-adherence summarised. A process for Decision-making after Pilot and feasibility Trials (ADePT) ${ }^{54}$ will be used to support systematic decisionmaking in moving forward with the trial.

\section{DISCUSSION}

The challenges anticipated include recruitment, a high level of attrition, poor literacy among participants and assessment across the wide age range of the 
study. Recruitment is a particular concern as refugee children and adolescents report feelings of mistrust and self-protection when in a setting that requires selfdisclosure, ${ }^{55}$ such as mental health interventions like TRT, which is likely to affect intervention and trial participation. However, the trial has been designed to mitigate these challenges where possible (eg, over-recruitment; retention strategies; in-person data collection) and will be instrumental in building the Swedish evidence base for refugee child mental health interventions. In particular, it will examine the impact of a brief intervention (weekly sessions over 7 weeks) with accompanied refugee children who are reporting symptoms of PTSD. The project also offers the opportunity to demonstrate that the use of RCTs to evaluate social interventions in real world, community settings is both achievable and valid.

\section{ETHICS AND DISSEMINATION}

The study was approved by the Regional Ethical Review Board in Uppsala (Ref. 2018/382) (24 ${ }^{\text {th }}$ February 2019). Any proposed changes to the protocol will be communicated to the Ethics Committee and approval will be sought before proceeding. Any modifications will be added to ISRCTN and communicated to relevant parties. The child and parent(s) will be informed of their right to withdraw from the research study at any time without giving a reason. All data collection relating to this case would then cease. All previously collected data relating to this child will still stand unless a child/parent also asks for all this to be removed from the data set (children/ legal guardians will be informed that this is possible up to the point that the data is analysed). All participants will be assured that there will be no adverse consequences of withdrawing from the study. Children will be able to receive the TRT programme regardless of whether they withdraw their involvement in the research study. The child and parent will be informed that data provided for the purpose of the trial will be treated confidentially. They will be made aware that in published reports the results will be reported anonymously and at a group level, meaning that it will not be possible to identify any individual or attribute any information to them. They will be informed that if they disclose anything concerning their personal safety then a safety protocol will be implemented. The results from the ASsIST trial are due to be submitted for publication in September 2021. Authorship will be granted for substantive contributions to the design, conduct, interpretation and reporting of the ASsIST trial; the ultimate decision on authorship will be made by the Principal Investigator (AS). Publications will be open access. The data sets generated during the current study will be available from the Principal Investigator (AS) on reasonable request.

\section{Patient and public involvement}

The development of the ASsIST study design was supported by a group of Refugee Advisors (parents and youth). The advisors attended research design planning meetings and will continue to attend meetings throughout the duration of the study. The advisors made several important contributions to the research development, including: (i) randomisation method; (ii) recruitment strategy; (iii) addressing different backgrounds of refugee youth in introduction sessions; and (iv) further testing of the validity of a suggested outcome measure. Specifically, the Refugee Advisors likened individual randomisation to the 'lottery of asylum' and indicated the uncertainty regarding whether they would receive the intervention with the others in the screening group (if screened in a group setting) would give rise to anxiety; it was more favourable to be randomised as a group.

\section{Trial status}

Protocol version 2 ( $1^{\text {st }}$ September 2019), recruitment efforts began in October 2019 and randomisation began on $24^{\text {th }}$ October 2019. Recruitment will continue until February 2021.

\section{Author affiliations}

${ }^{1}$ Child Health and Parenting, Department of Public Health and Caring Sciences, Uppsala University, Uppsala, Sweden

2Department of Psychology, Mid Sweden University, Östersund, Sweden ${ }^{3}$ Division of Clinical Psychology, The University of Manchester, Manchester, UK ${ }^{4}$ Division of Mental Health, Norwegian Institute of Public Health, Oslo, Norway ${ }^{5}$ Department of Pediatrics, University of Utah, Salt Lake City, Utah, USA

Acknowledgements The authors gratefully acknowledge the support of The Children and War Foundation and BRIS.

Contributors All authors contributed. AS is the Principal Investigator and led on the application for funding. GW is the project lead and led on the trial design, ethics submission, trial registration and writing the manuscript. $A B, R C, B 0$ and $\mathrm{BK}$ are trial advisers. ND, RSa, KF, FO, ER and EW are researchers on the trial team. EL and ZB are research assistants on the trial team. SGL, APA and AL are students leading concurrent projects. RSs will support the statistical analysis. IF and FS will lead on the economic analysis. All authors contributed to drafting and refining the study protocol and approved the final manuscript. Organisational structure and responsibilities: The Principal Investigator (AS) and lead researcher (GW) are responsible for the design and conduct of ASsIST; the preparation of the protocol and revisions; organising Steering Committee meetings; and publication of trial reports. The Steering Committee is formed of all other authors and patient and public involvement (PPI) representatives, referred to as Refugee Advisors. The Steering Committee is responsible for reviewing the progress of the study and, if necessary, agreeing changes to the protocol to facilitate the smooth running of the trial. A Data Management Committee has not been formed and no independent auditing will take place. Interim data monitoring will take place to inform aspects of trial conduct, such as recruitment. For instance, an internal pilot will be conducted (see Internal pilot section for more details). The ultimate decision on any amendments to the trial protocol or conduct will be made by the Principal Investigator (AS).

Funding The research is funded by the Kavli Trust (Grant: ID: A-321629). The funder has had no involvement in the design of the study or the writing of the manuscript, and will have no involvement in the collection, analysis and interpretation of data. Trial sponsor: Uppsala University. Box 256, Uppsala, 751 05, Sweden.

Competing interests None declared.

Patient and public involvement Patients and/or the public were involved in the design, or conduct, or reporting, or dissemination plans of this research. Refer to the Methods section for further details.

Patient consent for publication Not required.

Provenance and peer review Not commissioned; externally peer reviewed. 
Open access This is an open access article distributed in accordance with the Creative Commons Attribution Non Commercial (CC BY-NC 4.0) license, which permits others to distribute, remix, adapt, build upon this work non-commercially, and license their derivative works on different terms, provided the original work is properly cited, appropriate credit is given, any changes made indicated, and the use is non-commercial. See: http://creativecommons.org/licenses/by-nc/4.0/.

Author note GW, ND, RSa, KF, F0 and FS are researchers at CHAP, Uppsala University. EL and ZB are research assistants at CHAP, Uppsala University. SGL and RSs are doctoral students at CHAP, Uppsala University. APA is a Masters student at CHAP, Uppsala University. IF is a senior researcher in health economics at CHAP, Uppsala University. AB, ER and EW are researchers at the Department of Psychology and Social Work, Mid Sweden University. AL is a doctoral student at Mid Sweden University. RC is emeritus professor in child and family psychology at the University of Manchester. BO is senior researcher in the Child Health and Development Department at the Norwegian Institute of Public Health. BK is a clinician researcher in the Division of Child Protection and Family Health at the University of Utah and Safe and Healthy Families at Primary Children's Hospital. AS is a professor in social medicine and the Child Health and Parenting (CHAP) research group leader at Uppsala University.

\section{ORCID iDs}

Georgina Warner http://orcid.org/0000-0002-7850-9136

Filipa Sampaio http://orcid.org/0000-0002-5540-9853

\section{REFERENCES}

1 Swedish Migration Agency. Applications for asylum received, 2015. Available: www.migrationsverket.se/English/About-the-MigrationAgency/Statistics/Asylum

2 Attanayake V, McKay R, Joffres M, et al. Prevalence of mental disorders among children exposed to war: a systematic review of 7,920 children. Med Confl Surviv 2009;25:4-19.

3 Robjant K, Hassan R, Katona C. Mental health implications of detaining asylum seekers: systematic review. Br J Psychiatry 2009;194:306-12.

4 Belhadj Kouider E, Koglin U, Petermann F. Emotional and behavioral problems in migrant children and adolescents in Europe: a systematic review. Eur Child Adolesc Psychiatry 2014;23:373-91.

5 Oppedal B, Idsoe T. The role of social support in the acculturation and mental health of unaccompanied minor asylum seekers. Scand $\mathrm{J}$ Psychol 2015;56:203-11.

6 Sarkadi A, Ådahl K, Stenvall E, et al. Teaching recovery techniques: evaluation of a group intervention for unaccompanied refugee minors with symptoms of PTSD in Sweden. Eur Child Adolesc Psychiatry 2018;27:467-79.

7 Tam SY, Houlihan S, Melendez-Torres GJ. A systematic review of longitudinal risk and protective factors and correlates for posttraumatic stress and its natural history in Forcibly displaced children. Trauma Violence Abuse 2017;18:377-95.

8 Vervliet M, Lammertyn J, Broekaert E, et al. Longitudinal follow-up of the mental health of unaccompanied refugee minors. Eur Child Adolesc Psychiatry 2014;23:337-46.

9 Soykoek S, Mall V, Nehring I, et al. Post-Traumatic stress disorder in Syrian children of a German refugee cAMP. Lancet 2017;389:903-4.

10 Oppedal B, Solhaug AK, Fribor O, et al. Implementering OG evaluering AV teaching recovery techniques I asylmottak OG bosettingskommuner I Norge. Oslo, Norway: Norwegian Institute of Public Health, 2019.

11 Smith P, Dyregrov A, Yule W. Children and disaster: teaching recovery techniques. Bergen, Norway: Children and War Foundation, 2002.

12 Yule W, Dyregrov A, Raundalen M, et al. Children and war: the work of the children and war Foundation. Eur J Psychotraumatol 2013;4:18424.

13 Barron IG, Abdallah G, Smith P. Randomized control trial of a CBT trauma recovery program in Palestinian schools. Journal of Loss and Trauma 2013:18:306-21.

14 Barron I, Abdallah G, Heltne U. Randomized control trial of teaching recovery techniques in rural occupied Palestine: effect on adolescent dissociation. J Aggress Maltreat Trauma 2016;25:955-73.

15 Punamäki R-L, Peltonen K, Diab M, et al. Psychosocial interventions and emotion regulation among war-affected children: randomized control trial effects. Traumatology 2014;20:241-52.

16 Ooi CS. The efficacy and social validity of a group cognitive behavioural therapy for young migrants from war-affected countries (Doctoral dissertation, Curtin University) 2013.

17 Sarkadi A, Warner G, Salari R, et al. Evaluation of the teaching recovery techniques community-based intervention for unaccompanied refugee yOuth experiencing post-traumatic stress symptoms (Swedish unaccompanied yOuth refugee trial; support): study protocol for a randomised controlled trial. Trials 2020;21:63.

18 Perrin S, Meiser-Stedman R, Smith P. The children's revised impact of event scale (cries): validity as a screening instrument for PTSD. Behav Cogn Psychother 2005;33:487-98.

19 Edwards P. Questionnaires in clinical trials: guidelines for optimal design and administration. Trials 2010;11:2.

20 Keeshin BR, Strawn JR. Psychological and pharmacologic treatment of youth with posttraumatic stress disorder. Child Adolesc Psychiatr Clin N Am 2014;23:399-411.

21 Slone M, Mann S. Effects of war, terrorism and armed conflict on young children: a systematic review. Child Psychiatry Hum Dev 2016;47:950-65.

22 Feldman R, Vengrober A, Eidelman-Rothman M, et al. Stress reactivity in war-exposed young children with and without posttraumatic stress disorder: relations to maternal stress hormones, parenting, and child emotionality and regulation. Dev Psychopathol 2013;25:943-55.

23 Pfefferbaum B, Noffsinger MA, Jacobs AK, et al. Children's cognitive functioning in disasters and terrorism. Curr Psychiatry Rep 2016;18:48

24 Verlinden E, van Meijel EPM, Opmeer BC, et al. Characteristics of the children's revised impact of event scale in a clinically referred Dutch sample. J Trauma Stress 2014;27:338-44.

25 Salari R, Malekian C, Linck L, et al. Screening for PTSD symptoms in unaccompanied refugee minors: a test of the CRIES- 8 questionnaire in routine care. Scand J Public Health 2017;45:605-11.

26 Kroenke K, Spitzer RL, Williams JB. The PHQ-9: validity of a brief depression severity measure. J Gen Intern Med 2001;16:606-13.

27 Gilbody S, Richards D, Brealey S, et al. Screening for depression in medical settings with the patient health questionnaire (PHQ): a diagnostic meta-analysis. J Gen Intern Med 2007;22:1596-602.

28 Löwe B, Unützer J, Callahan CM, et al. Monitoring depression treatment outcomes with the patient health questionnaire-9. Med Care 2004;42:1194-201.

29 Richardson LP, McCauley E, Grossman DC, et al. Evaluation of the patient health Questionnaire-9 item for detecting major depression among adolescents. Pediatrics 2010;126:1117-23.

30 Spitzer RL, Kroenke K, Williams JBW, et al. A brief measure for assessing generalized anxiety disorder: the GAD-7. Arch Intern Med 2006;166:1092-7.

31 Kroenke K, Spitzer RL, Williams JBW, et al. Anxiety disorders in primary care: prevalence, impairment, comorbidity, and detection. Ann Intern Med 2007;146:317-25.

32 Löwe B, Decker O, Müller S, et al. Validation and standardization of the generalized anxiety disorder screener (GAD-7) in the general population. Med Care 2008;46:266-74.

33 Goodman R. The strengths and difficulties questionnaire: a research note. J Child Psychol Psychiatry 1997;38:581-6.

34 Goodman R. Psychometric properties of the strengths and difficulties questionnaire. J Am Acad Child Adolesc Psychiatry 2001;40:1337-45.

35 Schwarzer R, Jerusalem M. The general self-efficacy scale (GSE). Anxiety, Stress, and Coping 2010;12:329-45.

36 Scholz U, Doña BG, Sud S, et al. Is General self-efficacy a universal construct? Psychometric findings from 25 countries. Eur J Psychol Assess 2002;18:242.

37 Cantril H. Pattern of human concerns, 1965.

38 Mazur J, Szkultecka-Dębek M, Dzielska A, et al. What does the Cantril ladder measure in adolescence? Arch Med Sci 2018;14:182-9.

39 Sigvardsdotter E, Nilsson H, Malm A, et al. Development and Preliminary Validation of Refugee Trauma History Checklist (RTHC)-A Brief Checklist for Survey Studies. Int J Environ Res Public Health 2017;14:1175.

40 Stevens K. Developing a descriptive system for a new preferencebased measure of health-related quality of life for children. Qual Life Res 2009;18:1105-13.

41 Stevens K. Assessing the performance of a new generic measure of health-related quality of life for children and refining it for use in health state valuation. Appl Health Econ Health Policy 2011;9:157-69.

42 Stevens K. Valuation of the child health utility 9D index. Pharmacoeconomics 2012;30:729-47.

43 Stevens K, Ratcliffe J. Measuring and valuing health benefits for economic evaluation in adolescence: an assessment of the practicality and validity of the child health utility 9D in the Australian adolescent population. Value Health 2012;15:1092-9.

44 Chen G, Flynn T, Stevens K, et al. Assessing the health-related quality of life of Australian adolescents: an empirical comparison of 
the child health utility $9 \mathrm{D}$ and $\mathrm{EQ}-5 \mathrm{D}-\mathrm{Y}$ instruments. Value Health 2015;18:432-8.

45 Ratcliffe J, Stevens K, Flynn T, et al. An assessment of the construct validity of the CHU9D in the Australian adolescent general population. Qual Life Res 2012;21:717-25.

46 Bouwmans C, De Jong K, Timman R, et al. Feasibility, reliability and validity of a questionnaire on healthcare consumption and productivity loss in patients with a psychiatric disorder (TiC-P). BMC Health Serv Res 2013;13:217.

47 Posner K, Brown GK, Stanley B, et al. The Columbia-Suicide severity rating scale: initial validity and internal consistency findings from three multisite studies with adolescents and adults. Am J Psychiatry 2011;168:1266-77.

48 Hewitt CE, Torgerson DJ, Miles JNV. Is there another way to take account of noncompliance in randomized controlled trials? CMAJ 2006;175:347.

49 Jacobson NS, Truax P. Clinical significance: a statistical approach to defining meaningful change in psychotherapy research. $J$ Consult Clin Psychol 1991;59:12-19.
50 Evans C, Margison F, Barkham M. The contribution of reliable and clinically significant change methods to evidence-based mental health. Evid Based Ment Health 1998;1:70-2.

51 Barber J, Thompson S. Multiple regression of cost data: use of generalised linear models. J Health Serv Res Policy 2004;9:97-204.

52 Drummond MF, Sculpher MJ, Claxton K, et al. Methods for the economic evaluation of health care programmes. Oxford, UK: Oxford University Press, 2015

53 Whitehead AL, Julious SA, Cooper CL, et al. Estimating the sample size for a pilot randomised trial to minimise the overall trial sample size for the external pilot and main trial for a continuous outcome variable. Stat Methods Med Res 2016;25:1057-73.

54 Bugge C, Williams B, Hagen S, et al. A process for decision-making after pilot and feasibility trials (ADEPT): development following a feasibility study of a complex intervention for pelvic organ prolapse. Trials 2013;14:353.

55 van Os ECCC, Zijlstra AEE, Knorth EJE, et al. Finding keys: a systematic review of barriers and facilitators for refugee children's disclosure of their life stories. Trauma Violence Abuse 2020;21:242-60. 